\title{
Early diuretic use and mortality in critically ill patients with vasopressor support: a propensity score-matching analysis
}

\author{
Yanfei Shen ${ }^{1 *}$ D, Weimin Zhang $^{2}$ and Yong Shen ${ }^{3}$
}

\begin{abstract}
Background: The effect of loop diuretic use in critically ill patients on vasopressor support or in shock is unclear. This study aimed to explore the relationship between loop diuretic use and hospital mortality in critically ill patients with vasopressor support.

Methods: Data were extracted from the Medical Information Mart for Intensive Care III database. Adult patients with records of vasopressor use within $48 \mathrm{~h}$ after intensive care unit admission were screened. Multivariable logistic regression and propensity score matching was used to investigate any association.

Results: Data on 7828 patients were included. The crude hospital mortality was significantly lower in patients with diuretic use $(166 / 1469$ vs. $1171 / 6359, p<0.001)$. In the extended multivariable logistic models, the odds ratio (OR) of diuretic use was consistently significant in all six models (OR range $0.56-0.75, p<0.05$ for all). In the subgroup analysis, an interaction effect was detected between diuretic use and fluid balance (FB). In the positive FB subgroup, diuretic use was significantly associated with decreased mortality (OR 0.64, 95\% confidence interval (Cl) $0.51-0.78$ ) but was insignificant in the negative FB subgroup. In the other subgroups of mean arterial pressure, maximum sequential organ failure assessment score, and lactate level, the association between diuretic use and mortality remained significant and no interaction was detected. After propensity score matching, 1463 cases from each group were well matched. The mortality remained significantly lower in the diuretic use group (165/1463 vs. 231/1463, $p<0.001$ ).
\end{abstract}

Conclusions: Although residual confounding cannot be excluded, loop diuretic use is associated with lower mortality.

Keywords: Critical care, Loop diuretics, Vasopressor, Mortality, Fluid balance

\section{Background}

Optimizing fluid status is fundamental in critical care but is challenging to achieve. Sufficient fluid resuscitation is crucial in stabilizing the hemodynamic status of patients, and largely depends on large-volume fluid administration. Additionally, a positive correlation between fluid overload and adverse outcomes has been reported in various diseases, such as sepsis $[1,2]$, acute lung injury [3], and acute kidney injury (AKI) [4].

Loop diuretics are commonly prescribed to alleviate fluid overload. However, their efficacy and safety remain unclear. Several studies have shown that diuretics may induce

\footnotetext{
* Correspondence: snow.shen@hotmail.com

'Department of Intensive Care Unit, Zhejiang Hospital, No. 12, Lingyin Road, Hangzhou, Zhejiang 322100, People's Republic of China

Full list of author information is available at the end of the article
}

oxidative stress and further aggravate renal injury in patients with severe AKI $[5,6]$. However, a pilot randomized controlled trial (RCT) failed to confirm any specific incremental risk with furosemide use in AKI patients [7]. Furthermore, Grams et al. found that diuretic therapy was associated with improved survival in AKI patients [8]. Despite these contradictory conclusions, loop diuretics are still widely used in clinical practice to increase urine output (UO) and reduce the rate of edema. In a large survey of critically ill patients, diuretics were prescribed in more than $50 \%$ of the population during intensive care unit (ICU) stay [9]. Another large online questionnaire survey also reported that furosemide was commonly used, especially in critically ill patients with positive fluid balance (FB) and acute pulmonary edema [10]. In clinical situations, a patient's hemodynamic status may become relatively stable with 
vasopressor support after adequate fluid resuscitation. One major concern is that diuretic use may induce hypovolemia and further compromise the hemodynamic status, and it remains unclear if diuretics can alleviate the detrimental effects of excessive fluid accumulation in these patients.

This study aimed to explore the relationship between diuretic use and hospital mortality in critically ill patients on vasopressor support, using logistic regression and propensity score matching (PSM). Subgroup analysis was also performed to investigate the interaction effect between diuretic use and other potential covariates.

\section{Methods}

\section{Database introduction}

All the data in the current study were extracted from an online international database- Medical Information Mart for Intensive Care III (MIMIC III) - that was published by the Massachusetts Institute of Technology, with approval from the review boards of the Massachusetts Institute of Technology and Beth Israel Deaconess Medical Center [11]. All the patients in the database were de-identified for privacy protection, and the need for informed consent was waived. One author (Y S) obtained access to this database (certification number 1564657) and was responsible for data extraction.

\section{Inclusion and exclusion criteria}

Adult patients with medical records indicating any vasopressor use within $48 \mathrm{~h}$ after ICU admission were initially screened. The recorded vasopressors included dobutamine, dopamine, norepinephrine, epinephrine, vasopressin, and phenylephrine. Patients who were younger than 18 years or spent less than $48 \mathrm{~h}$ in the ICU were excluded. For patients who were admitted to the ICU more than once, only the first ICU stay was considered.

\section{Data extraction}

Data on the demographic characteristics, comorbidities, laboratory outcomes, diuretic use, FB, mean arterial pressure (MAP), and disease severity score were extracted from the database. Diuretic use was defined as the use of any loop diuretic within $48 \mathrm{~h}$ after ICU admission, including furosemide, torasemide, and bumetanide. Vasopressor use was defined as the use of any vasopressor agent, including norepinephrine, epinephrine, dobutamine, dopamine, vasopressin, and phenylephrine, within $48 \mathrm{~h}$ after ICU admission.

\section{Stratification and outcome definition}

Subgroup analysis was performed to explore the possible interaction between diuretic use and hemodynamic indices as well as disease severity. Stratification was performed according to the FB status within $48 \mathrm{~h}$ after ICU admission ( $\geq$ or $<0 \mathrm{ml} / \mathrm{kg} / 48 \mathrm{~h}$ ), mean MAP ( $\geq$ or $<70 \mathrm{mmHg}$ ), median value of maximum sequential organ failure assessment (SOFA) score during ICU stay $(\geq$ or $<10$ ), and median value of maximum lactate level during ICU stay ( $\geq$ or $<2.7 \mathrm{mmol} / \mathrm{L}$ ). The primary endpoint was hospital mortality. AKI was defined as a 1.5-fold increase in serum creatinine $(\mathrm{sCr})$ level during the ICU stay relative to baseline $\mathrm{sCr}$ level, according to the creatinine-based Kidney Disease Improving Global Outcomes criteria, without urine output $[12,13]$. For patients without previous sCr data, it was estimated using the following formula [14]: $\mathrm{sCr}=0.74$ -0.2 (if female) +0.08 (if black) $+0.0039 \times$ age (in years)

\section{Propensity score matching}

PSM [15] was used to minimize the effect of confounding factors such as hemodynamic indices and disease severity, which may lead to outcome bias. The propensity score was assigned based on the probability that a patient would receive diuretic therapy and estimated using a multivariable logistic regression model. A one-to-one nearest neighbor matching algorithm was applied using a caliper width of 0.05 . The following variables were selected to generate the propensity score: age, weight, diabetes mellitus, hypertension, cardiac disease, AKI, SOFA score on ICU admission, white blood cells, fluid intake volume, and proportions of different vasopressors. Kernel density plots of the $p$ score were used to examine the PSM degree. Finally, 1463 matched pairs were generated and applied to further analyses.

\section{Management of missing data}

Variables with missing data are common in the MIMIC III database. For serum lactate and albumin values, more than $20 \%$ were missing and were removed from this analysis. For other continuous variables with missing values less than $5 \%$, the missing values were replaced by the mean or median values.

\section{Statistical analysis}

Continuous variables are expressed as mean \pm standard deviation or median (interquartile range), as appropriate. The student's $t$ test, analysis of variance, Wilcoxon rank-sum test, or Kruskal-Wallis test was used, as appropriate. Categorical data are expressed as proportions and compared using the $\chi^{2}$ test. An extended logistic model approach was used for covariate adjustment: as hemodynamic indices such as FB, MAP, and serum lactate level and disease severity are important factors affecting the decision to use diuretics in clinical practice, subgroup analyses were performed as described above. Multicollinearity was tested using the variance inflation factor (VIF) method, with a VIF $\geq 5$ indicating the presence of multicollinearity. Goodness of fit tests were applied to all logistic regression models. PSM was used to minimize the imbalance between groups. A two-tailed test was performed, and $p<0.05$ was 
considered statistically significant. All statistical analyses were performed using Stata 11.2 (Stata Corp., College Station, TX, USA).

\section{Results}

\section{Baseline characteristics}

Data on 7828 patients were included. The flow chart of patient selection is presented in Fig. 1. The comparisons of the baseline characteristics are listed in Table 1. The overall hospital mortality rate was $17.1 \%$. The difference in MAP was small between the groups $(74.6 \pm 8.2$ vs. 73.0 $\pm 7.1, p<0.001)$, and the trends of the average hourly MAP are presented in Fig. 2. The SOFA score on admission was also relatively similar in the diuretic use and no diuretic use groups (6 (4-8) vs. $6(4-9), p=0.012$ ). The fluid intake within $48 \mathrm{~h}$ was significantly higher in patients with no diuretic use $(113.8 \pm 77.1$ vs. $95.5 \pm 55.6$, $p<0.002)$ while the UO volume was similar across the groups despite statistical significance $(48.5 \pm 38.2$ vs. 51.2 $\pm 28.4, p=0.009$ ). However, the hospital mortality was significantly lower in patients with diuretic use (166/1469 vs. $1171 / 6359, p<0.001)$. Additional baseline information, including the doses and proportions of different vasopressors, is presented in Additional file 1 (Table S1).

\section{Association between diuretic use and hospital mortality} In the extended multivariable logistic models (Table 2), we observed that the odds ratio (OR) of diuretic use was consistently significant in all six models (OR range 0.56$0.75, p<0.05$ for all). Subgroup analysis was performed according to the FB, MAP, maximum SOFA score, and lactate level (Fig. 3). The OR of diuretic use was significant in the MAP subgroups ( $\geq 70 \mathrm{mmHg}$ : OR $0.70,95 \%$

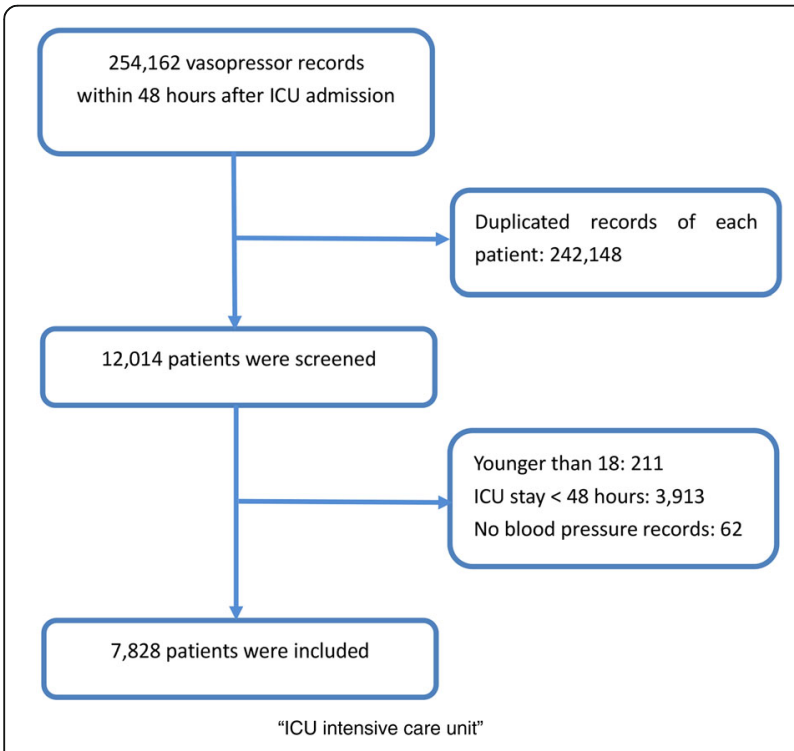

Fig. 1 Flow chart of patient selection from the MIMIC III database confidence interval (CI) $0.55-0.88, \quad p=0.002 ; \quad<70$ mmHg: OR $0.50,95 \%$ CI $0.36-0.70, p<0.001)$, SOFA score $(\geq 10$ : OR $0.63,95 \%$ CI $0.51-0.77, p<0.001 ;<10$ : OR $0.54,95 \%$ CI $0.33-0.86, p=0.010$ ), and lactate level $(\geq 2.7$ : OR $0.45,95 \%$ CI $0.35-0.57, p<0.001 ;<2.7$ : OR $0.69,95 \%$ CI $0.51-0.94, p=0.020$ ), and no significant interaction was observed. However, in the FB subgroups, an interactive effect was detected ( $p$ value for interaction, 0.038). In the positive FB group, diuretic use was significantly associated with decreased mortality (OR 0.64, 95\% CI 0.51$0.78, p<0.001)$, and insignificantly associated with it in the negative FB group (OR 0.73, 95\% CI 0.47-1.14, $p=0.170$ ).

\section{Outcomes after propensity score matching}

After PSM, 1463 cases from each group were well matched by a 1:1 matching algorithm (Table 3). The overall quality of the matched sample was assessed by comparing the standardized difference of the means and the ratio of the variances between the propensity scores of both groups as well as by graphically inspecting the propensity scores between the groups (Additional file 1: Figure S1). There was no significant difference between the two matched groups with regards to all twelve covariates, including fluid intake $(94.1 \pm 57.4$ vs. $95.6 \pm$ 55.7, $p=0.483$ ) and proportions of different vasopressors. Among the 1463 propensity-matched pairs, we found that the hospital mortality was significantly lower in the diuretic use group $(165 / 1463$ vs. $231 / 1463, p<0.001)$. The UO volume was significantly higher in the diuretic use group $(51.3 \pm 28.5$ vs. $44.5 \pm 28.6, p<0.001)$, and the mean MAP was comparable $(73.0 \pm 7.1$ vs. $74.5 \pm 7.8, p<0.001)$.

\section{Sensitivity analysis}

Vasopressin (despite weak recommendations in the sepsis guidelines) and phenylephrine are usually not used as first-line vasopressors. For example, some vasopressors may be used in patients during postoperative recovery from anesthesia, and vasopressin may be prescribed for gastrointestinal bleeding instead of septic shock. To test the robustness of our findings, we performed a sensitivity analysis excluding these two vasopressors. The outcomes remained stable after adjustment for confounders (Additional file 1: Table S2) and in the comparisons after PSM (Additional file 1: Table S3).

\section{Discussion}

The present study demonstrates that in critically ill patients with vasopressor use the use of diuretics is associated with significantly decreased hospital mortality. This result was robust in the PSM analysis after adjustment for covariates and remained consistent in the subgroups of MAP, lactate level, and SOFA score. Unexpectedly, an interaction was detected between FB and diuretic use. A potential benefit was only observed in patients with 
Table 1 Comparisons of the baseline characteristics between patients with and without diuretic use

\begin{tabular}{|c|c|c|c|c|}
\hline Variables & $\begin{array}{l}\text { All patients } \\
(n=7828)\end{array}$ & $\begin{array}{l}\text { No diuretic use } \\
(n=6359)\end{array}$ & $\begin{array}{l}\text { Diuretic use } \\
(n=1469)\end{array}$ & $P$ \\
\hline Age (years) & $67.0 \pm 14.2$ & $66.5 \pm 14.5$ & $70.0 \pm 12.3$ & $<0.001$ \\
\hline Male, $n(\%)$ & $4588(58.6)$ & $3749(58.9)$ & $839(57.1)$ & 0.196 \\
\hline Weight (kg) & $82.3 \pm 22.6$ & $81.7 \pm 22.3$ & $84.9 \pm 24.0$ & $<0.001$ \\
\hline Emergency, $n(\%)$ & $6072(77.5)$ & $5075(79.8)$ & $998(67.9)$ & $<0.001$ \\
\hline \multicolumn{5}{|l|}{ Comorbidities, n (\%) } \\
\hline Diabetes mellitus & $2390(30.5)$ & $1850(29.1)$ & $540(36.7)$ & $<0.001$ \\
\hline Hypertension & $3831(48.9)$ & $3025(47.5)$ & $806(54.8)$ & $<0.001$ \\
\hline Cardiac disease & $3431(43.8)$ & $2603(41.2)$ & $848(57.7)$ & $<0.001$ \\
\hline Acute or chronic heart failure & $2986(38.1)$ & $2329(36.6)$ & $657(44.7)$ & $<0.001$ \\
\hline Intracranial hemorrhage & $273(3.5)$ & $249(3.9)$ & $24(1.6)$ & $<0.001$ \\
\hline \multicolumn{5}{|l|}{ Fluid balance } \\
\hline Fluid intake (ml/kg/48 h) & $110.3 \pm 73.9$ & $113.8 \pm 77.1$ & $95.5 \pm 55.6$ & $<0.001$ \\
\hline Urine output (ml/kg/48 h) & $49.0 \pm 36.6$ & $48.5 \pm 38.2$ & $51.2 \pm 28.4$ & 0.009 \\
\hline Fluid balance (ml/kg/48 h) & $45.1 \pm 76.7$ & $49.9 \pm 79.7$ & $24.2 \pm 57.9$ & $<0.001$ \\
\hline \multicolumn{5}{|l|}{ MAP after ICU admission } \\
\hline MAP on ICU admission (mmHg) & $77.4 \pm 17.3$ & $77.7 \pm 17.6$ & $76.2 \pm 15.8$ & 0.002 \\
\hline Mean MAP (mmHg) & $74.3 \pm 8.1$ & $74.6 \pm 8.2$ & $73.0 \pm 7.1$ & $<0.001$ \\
\hline Maximum MAP (mmHg) & $115.6 \pm 40.1$ & $115.9 \pm 38.7$ & $114.4 \pm 45.5$ & 0.19 \\
\hline Minimum MAP (mmHg) & $48.2 \pm 14.7$ & $48.3 \pm 14.5$ & $47.7 \pm 15.3$ & 0.139 \\
\hline \multicolumn{5}{|l|}{ Disease severity scores, median (IQR) } \\
\hline SOFA score on ICU admission & $6(4-8)$ & $6(4-8)$ & $6(4-9)$ & 0.0122 \\
\hline Maximum SOFA score during ICU stay & $10(8-13)$ & $10(8-13)$ & $11(8-13)$ & 0.2377 \\
\hline SAPS II on ICU admission & $41(32-52)$ & $41(32-52)$ & $41(34-51)$ & 0.0151 \\
\hline GCS score on ICU admission & $7(3-14)$ & $8(3-14)$ & $4(3-11)$ & $<0.001$ \\
\hline Maximum GCS score during ICU stay & $15(15-15)$ & $15(15-15)$ & $15(15-15)$ & 0.001 \\
\hline \multicolumn{5}{|l|}{ Biochemical indices } \\
\hline Maximum serum creatinine level (mg/dl) & $1.87 \pm 1.75$ & $1.88 \pm 1.81$ & $1.83 \pm 1.45$ & 0.254 \\
\hline Maximum white blood cell count $\left(10^{9} / \mathrm{l}\right)$ & $18.5 \pm 11.5$ & $18.7 \pm 11.8$ & $17.5 \pm 9.9$ & $<0.001$ \\
\hline Maximum serum lactate level (mmol/l) & $3.7 \pm 3.2(n=6168)$ & $3.7 \pm 3.4(n=4799)$ & $3.5 \pm 2.7(n=1369)$ & 0.004 \\
\hline \multicolumn{5}{|l|}{ Oxygenation indexes } \\
\hline Mechanical ventilation, $n(\%)$ & $6646(84.9)$ & $5323(83.7)$ & $1323(90.0)$ & $<0.001$ \\
\hline Duration of mechanical ventilation (h) & $98.2 \pm 175.8$ & $102.9 \pm 181.8$ & $77.8 \pm 145.7$ & $<0.001$ \\
\hline $\mathrm{PO}_{2} / \mathrm{FiO}_{2}$ at ICU admission & $258.0 \pm 137.3$ & $264.1 \pm 140.2$ & $232.6 \pm 212.3$ & $<0.001$ \\
\hline Minimum $\mathrm{PO}_{2} / \mathrm{FiO}_{2}$ during ICU stay & $156.2 \pm 103.5$ & $154.8 \pm 104.5$ & $161.8 \pm 99.0$ & 0.029 \\
\hline \multicolumn{5}{|l|}{ Clinical outcomes } \\
\hline Hyponatremia, $n(\%)^{*}$ & 3100 (39.6) & $2433(38.2)$ & $667(45.4)$ & $<0.001$ \\
\hline Hypokalemia, $n(\%)^{*}$ & $2981(38.1)$ & $2475(38.9)$ & $506(34.4)$ & 0.001 \\
\hline Hypocalcemia, $n(\%)^{*}$ & $5569(71.1)$ & $4608(72.4)$ & $961(65.4)$ & $<0.001$ \\
\hline ICU LOS (days) & $7.5 \pm 8.5$ & $7.7 \pm 8.7$ & $6.7 \pm 7.4$ & $<0.001$ \\
\hline Hospital LOS (days) & $13.7 \pm 12.1$ & $13.9 \pm 12.6$ & $12.5 \pm 10.0$ & $<0.001$ \\
\hline $\mathrm{AKI}, n(\%)$ & $2358(30.1)$ & $1848(29.1)$ & $510(34.7)$ & $<0.001$ \\
\hline AKI stage $3, n(\%)$ & $1108(14.1)$ & $917(14.4)$ & $191(13.0)$ & 0.160 \\
\hline
\end{tabular}


Table 1 Comparisons of the baseline characteristics between patients with and without diuretic use (Continued)

\begin{tabular}{|c|c|c|c|c|}
\hline Variables & $\begin{array}{l}\text { All patients } \\
(n=7828)\end{array}$ & $\begin{array}{l}\text { No diuretic use } \\
(n=6359)\end{array}$ & $\begin{array}{l}\text { Diuretic use } \\
(n=1469)\end{array}$ & $P$ \\
\hline Dialysis, $n$ (\%) & $680(8.7)$ & $561(8.8)$ & $119(8.1)$ & 0.376 \\
\hline Hospital mortality, n (\%) & $1337(17.1)$ & $1171(18.4)$ & $166(11.3)$ & $<0.001$ \\
\hline
\end{tabular}

Values are shown as mean \pm standard deviation unless otherwise indicated

AKI acute kidney injury, GCS Glasgow Coma Scale, ICU intensive care unit, IQR interquartile range, LOS length of stay, MAP mean arterial pressure, SAPS I/ Simplified Acute Physiology Score II, SOFA sequential organ failure assessment

* These three electrolyte disturbances were diagnosed according to the electrolyte value within $48 \mathrm{~h}$ after ICU admission

positive FB; this benefit was insignificant in the negative FB subgroup. Our findings are suggestive of a possible beneficial role for diuretics in patients with vasopressor use, which has not been previously demonstrated.

Adequate fluid resuscitation is fundamental in critical care $[16,17]$, and entails aggressive large-volume fluid intake. However, several studies have reported that positive FB was strongly associated with worse outcomes [18], such as respiratory dysfunction [3], high intra-abdominal pressure [19], coagulation disorder [20], and increased mortality [21]. Excessive fluid accumulation may impair clinical outcomes. Several studies have demonstrated that strategies aimed at avoiding an excessive positive FB such as restricted fluid resuscitation may be beneficial. One systematic review found that restricted fluid resuscitation was associated with decreased mortality in trauma patients compared with initial liberal fluid resuscitation [22]. Silversides et al. also reported that in patients with acute respiratory distress syndrome or sepsis a conservative fluid strategy resulted in a shorter duration of ventilator use and ICU stay [23]. Thus, the use of strategies aimed at alleviating fluid overload following initial fluid resuscitation has become very important in ensuring better prognosis, suggesting the potential role of diuretic use. However, in contrast to the established knowledge on fluid accumulation, conclusions on the effectiveness and safety of loop diuretics in critically ill patients remain controversial.

In a pharmacoepidemiologic evaluation [24], furosemide was listed as a nephrotoxic drug. Mehta et al. investigated data from 552 AKI patients and reported that diuretic use was significantly associated with higher mortality and

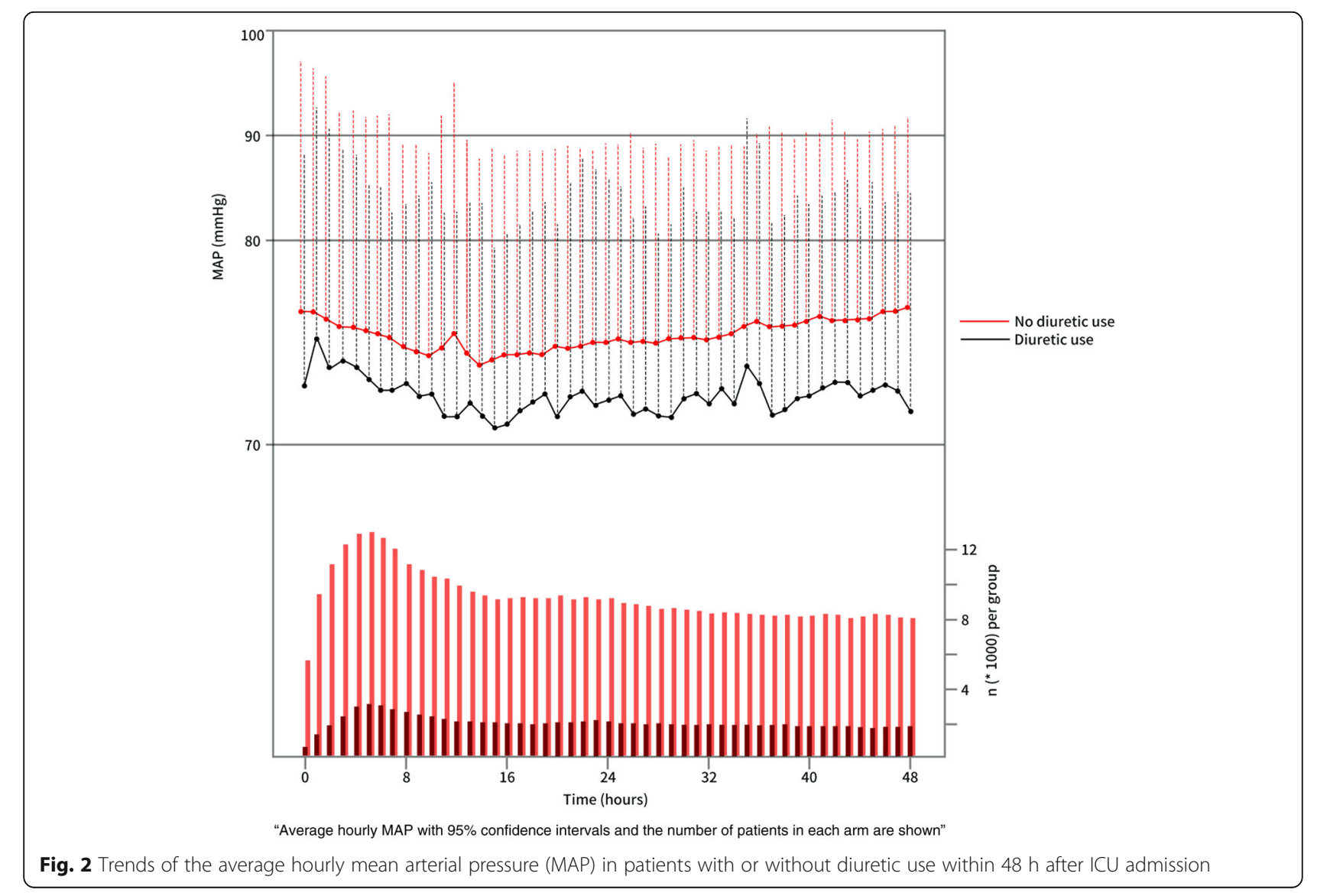


Table 2 Association between diuretic use and hospital mortality using an extended model approach

\begin{tabular}{llll}
\hline & Odds ratio of diuretic use & $95 \%$ confidence interval & $P$ \\
\hline Model 1 & 0.56 & $(0.47-0.67)$ & $<0.001$ \\
Model 2 & 0.62 & $(0.51-0.74)$ & $<0.001$ \\
Model 3 & 0.58 & $(0.48-0.71)$ & $<0.001$ \\
Model 4 & 0.69 & $(0.55-0.81)$ & $<0.001$ \\
Model 5 & 0.75 & $(0.62-0.91)$ & 0.004 \\
Model 5 & 0.69 & $(0.57-0.84)$ & $<0.001$
\end{tabular}

Adjusted covariates: Model $1=$ diuretic use. Model $2=$ Model

$1+$ (comorbidities including intracranial hemorrhage, hypertension, acute kidney injury, coronary disease and diabetes). Model $3=$ Model $2+$ (gender, age, biochemical indices including white blood cell count and SOFA score on ICU admission). Model $4=$ Model $3+$ (fluid balance within $48 \mathrm{~h}$ after intensive care unit admission). Model $5=$ Model $4+$ (proportion of patients receiving vasopressors including norepinephrine, epinephrine, dopamine, and dobutamine). Model $6=$ Model $4+$ (dose of vasopressors including norepinephrine, epinephrine, dopamine, and dobutamine)

Note: The mean variance inflation factor was 2.58 and 2.42 and the $p$ value of the goodness of fit was 0.99 and 0.99 for Model 5 and Model 6, respectively

worse renal function [25]. In critically ill patients requiring acute dialysis post-surgery, a higher accumulative diuretic dose was significantly associated with increased mortality [26]. However, another multicenter, observational study reported that diuretics were commonly prescribed in 1743 patients with acute renal failure, and that their use was not associated with deteriorated outcomes, including mortality [27]. A small pilot RCT also failed to confirm any specific incremental risk with furosemide use in AKI patients, but suggested an insignificant downward trend in the 90 -day mortality (21.6\% vs. $30.5 \%$ ) [7].
On reviewing all the aforementioned studies, we speculated that the risk of death or other poor outcomes was confounded by AKI severity (baseline $\mathrm{sCr}$ levels of $3.8 \mathrm{mg} / \mathrm{dl} \mathrm{[25],} 3.3 \mathrm{mg} / \mathrm{dl}$ [26], $2.0 \mathrm{mg} / \mathrm{dl} \mathrm{[27],} \mathrm{and}$ $1.8 \mathrm{mg} / \mathrm{dl}$ [7]). Diuretic use in patients with severe AKI may contribute to unfavorable outcomes. Grams et al. concluded that post-AKI diuretic therapy was associated with improved 60-day survival in patients with relatively mild AKI (baseline sCr level $1.6 \mathrm{mg} / \mathrm{dl}$ ) [8]. In our study, the mean baseline $\mathrm{sCr}$ level was $1.39 \mathrm{mg} / \mathrm{dl}$ and diuretic use was also associated with lower hospital mortality. Additionally, we speculated that the association between diuretic use and mortality might be mediated by fluid status. In the current study, the UO volumes in the two groups were similar and both reached the specific therapeutic end goals ( $\mathrm{UO}>1 \mathrm{ml} / \mathrm{kg} / \mathrm{h}[28]$ ) after adequate resuscitation. Meanwhile, the fluid intake volume was significantly higher in the no diuretic use group, which directly lead to a higher positive FB. In clinical practice, high fluid intake is one common strategy to reach resuscitation goals, including UO volume. However, due to the retrospective nature of the study, we could not infer if the UO volume impacted the fluid administration protocol decision. After adjustment for fluid intake using PSM, the FB was still significantly lower in the diuretic use group and largely depended on the increased UO; this was more significant in the sensitivity analysis. To a certain extent, this speculation also explained why this association was insignificant or even opposite in

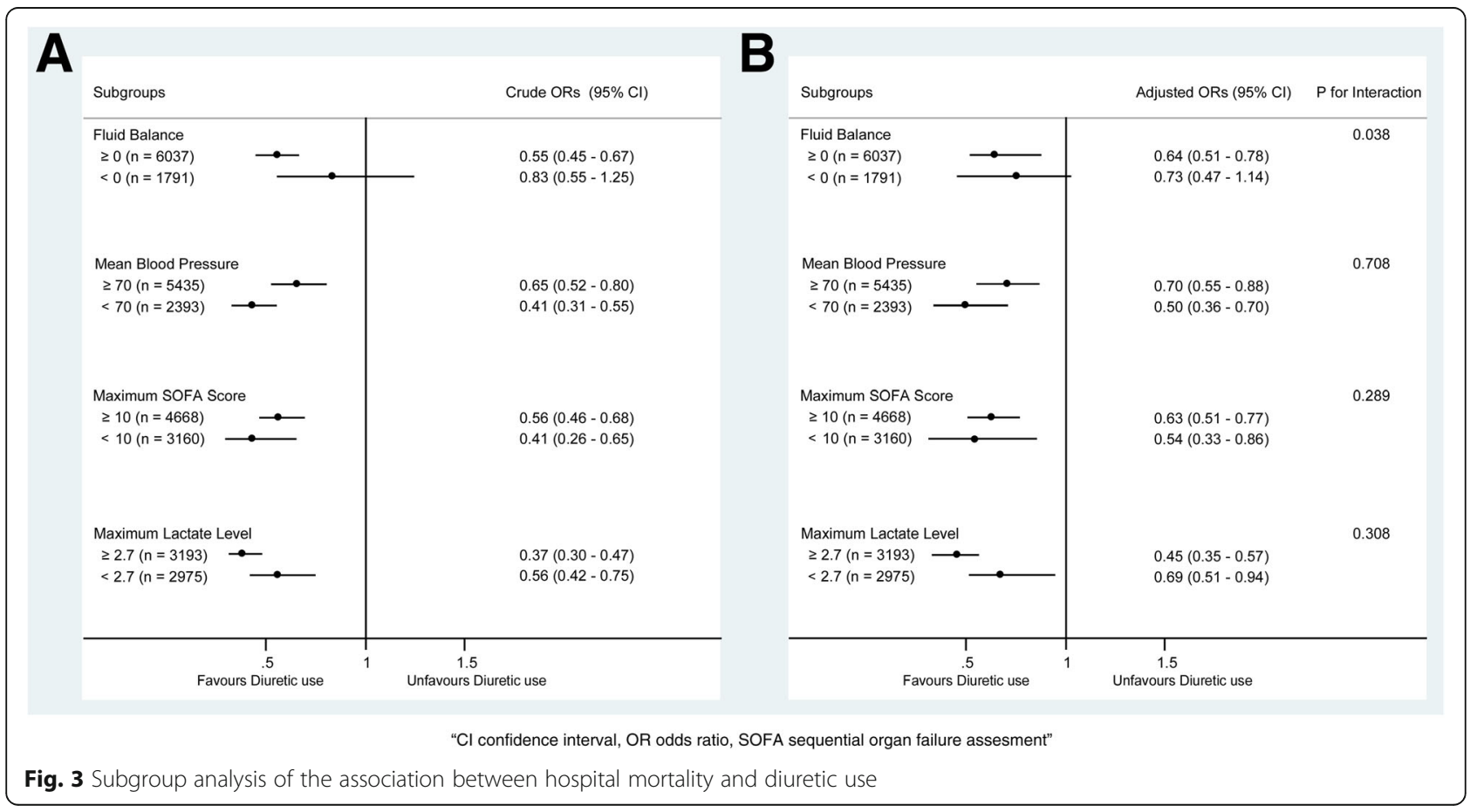


Table 3 Comparisons of the covariates after propensity score matching

\begin{tabular}{|c|c|c|c|}
\hline Variables & $\begin{array}{l}\text { No diuretic use } \\
(n=1463)\end{array}$ & $\begin{array}{l}\text { Diuretic use } \\
(n=1463)\end{array}$ & $P$ \\
\hline Age (years) & $70.3 \pm 11.9$ & $69.9 \pm 12.3$ & 0.472 \\
\hline Weight (kg) & $84.4 \pm 23.1$ & $84.9 \pm 24.0$ & 0.599 \\
\hline Diabetes mellitus, $n$ (\%) & $514(35.2)$ & $535(36.5)$ & 0.418 \\
\hline Hypertension, $n(\%)$ & $820(53.5)$ & $802(54.8)$ & 0.503 \\
\hline Cardiac disease, $n(\%)$ & $829(58.5)$ & $842(57.5)$ & 0.627 \\
\hline $\mathrm{AKI}, n(\%)$ & $496(34.1)$ & $504(34.4)$ & 0.755 \\
\hline SOFA score on ICU admission, median (IQR) & $6(4-8)$ & $6(4-9)$ & 0.999 \\
\hline Maximum white blood cell count $\left(10^{9} / l\right)$ & $17.0 \pm 7.6$ & $17.5 \pm 9.9$ & 0.198 \\
\hline Fluid intake (ml/kg/48 h) & $94.1 \pm 57.4$ & $95.6 \pm 55.7$ & 0.483 \\
\hline Norepinephrine (mg/kg/48 h) & $0.053 \pm 0.139$ & $0.052 \pm 0.151$ & 0.814 \\
\hline Dopamine (mg/kg/48 h) & $0.782 \pm 3.054$ & $0.843 \pm 3.665$ & 0.623 \\
\hline Dobutamine (mg/kg/48 h) & $0.193 \pm 1.118$ & $0.238 \pm 1.789$ & 0.411 \\
\hline Epinephrine (mg/kg/48 h) & $0.008 \pm 0.125$ & $0.009 \pm 0.084$ & 0.676 \\
\hline Vasopressin (mg/kg/48 h) & $0.071 \pm 0.353$ & $0.080 \pm 0.359$ & 0.495 \\
\hline Mechanical ventilation & 1317 & 1317 & 1.000 \\
\hline \multicolumn{4}{|l|}{ Clinical outcomes } \\
\hline Hospital mortality, $n(\%)$ & $231(15.6)$ & $165(11.2)$ & $<0.001$ \\
\hline Urine output (ml/kg/48 h) & $44.5 \pm 28.6$ & $51.3 \pm 28.5$ & $<0.001$ \\
\hline Fluid balance (ml/kg/48 h) & $35.3 \pm 53.8$ & $24.3 \pm 58.1$ & $<0.001$ \\
\hline Mean MAP (mmHg) & $74.5 \pm 7.8$ & $73.0 \pm 7.1$ & $<0.001$ \\
\hline
\end{tabular}

Values are shown as mean \pm standard deviation unless otherwise indicated

$A K I$ acute kidney injury, ICU intensive care unit, IQR interquartile range, MAP mean arterial pressure, SOFA sequential organ failure assessment

patients with severe AKI, as they may fail to respond to the diuretic challenge.

Furthermore, an interaction between FB and diuretic use was detected, and the benefit associated with diuretic use existed only in the positive FB subgroup; it remained insignificant in the negative FB subgroup. This finding further supports our speculation that in patients with a positive FB, the use of diuretics may alleviate fluid accumulation and thus contribute to improved survival. However, compared with achieving a mildly negative FB, achieving a greater degree of negative FB could not further reduce mortality in critically ill patients [29]. Thus, the use of loop diuretics may further increase the negative FB volume; however, this was not conducive to survival in the negative FB subgroup.

One major concern is that diuretic use may induce hypovolemia and compromise the already impaired hemodynamic status in such patients. In the current study, we found a significant difference in the MAP between the two groups, which could be affected by less fluid accumulation in the diuretic group. However, the clinical difference was relatively small both in the crude comparison and in the comparison after PSM (2$3 \mathrm{mmHg}$ ). This finding is consistent with the conclusion of Yeh et al. that furosemide in critically ill trauma patients resulted in lower fluid accumulation, with no detrimental effect on the hemodynamic parameters [30]. This finding was also supported by the results of a study by Shann [31]. Furthermore, we noticed that the association between diuretic use and hospital mortality was still significant in the subgroups with high SOFA scores, high maximum lactate levels, and low MAP, which suggested the feasibility of the appropriate use of loop diuretics in these patients.

This study has several limitations. First, there are many confounders for diuretic use in clinical practice, such as the reason for ICU admission, disease severity, kidney function, hemodynamic status, vasopressors, and clinician preference. In the current study, we included as many confounders as possible to minimize potential bias. However, due to the retrospective nature, some information was unavailable in this database. Rigorously designed randomized controlled studies may be the only solution for the imbalance between these two groups. Second, we inferred that the use of loop diuretics might reduce the mortality in this specific cohort. However, in addition to their diuretic effect, the adverse effects of loop diuretics, such as renal oxidative stress [5], may be overridden by the benefit of reduced positive FB. Caution should be exercised in interpreting these findings, especially in patients without fluid accumulation or those with severe 
AKI. Third, despite the benefit of vasopressors revealed in the current study, the inter-heterogeneity of included patients was still a concern. For instance, is the effect of diuretics consistent in patients with slight or massive vasopressor support? More studies are needed to verify this interaction. Fifth, missing data is an important issue in the analysis. In the current study, the percentage of missing values of most variables is relatively small. Thus, these missing values were replaced by their mean or median values instead of using multiple imputation methods. Finally, the causal relationship between diuretic use and mortality could not be confirmed. While the use of a propensity score can further support our speculation, it still cannot overcome the primary limitation associated with the observational nature of the study. Further prospective studies are needed to verify our hypothesis.

\section{Conclusions}

Diuretic use was associated with reduced hospital mortality in critically ill patients with vasopressor support, without an obvious compromise in the MAP. This association was not affected by disease severity and serum lactate levels; however, this was only significant in patients with a positive FB. Future larger randomized clinical trials are required to confirm and validate this association.

\section{Additional file}

Additional file 1: Figure $\mathbf{S 1}$. Kernel density plots of the propensity score before and after propensity score matching. Table S1. Comparisons of the baseline characteristics between patients with and without diuretic use. Table S2. Association between diuretic use and hospital mortality using an extended model approach $(n=4747)$. Table S3. Comparisons of covariates after propensity score matching. Table S4. Comparisons between subgroups with positive and negative fluid balance within $48 \mathrm{~h}$ after ICU admission. (DOCX $273 \mathrm{~kb}$ )

\section{Abbreviations}

AKI: Acute kidney injury; Cl: Confidence interval; FB: Fluid balance; ICU: Intensive care unit; MAP: Mean arterial pressure; MIMIC III: Medical Information Mart for Intensive Care III; OR: Odds ratio; PSM: Propensity score matching; RCT: Randomized controlled trial; sCr: Serum creatinine; SOFA: Sequential organ failure assessment; UO: Urine output; VIF: Variance inflation factor

\section{Acknowledgements}

We thank Dr. Jing Yan (Zhejiang Hospital, Hangzhou, China) and Guolong Cai (Zhejiang Hospital, Hangzhou, China) for their help in this revision.

\section{Funding}

Not applicable.

\section{Availability of data and materials}

The datasets presented in the current study are available in the MIMIC III database (https://physionet.org/works/MIMICIIIClinicalDatabase/files/).

\section{Authors' contributions}

YaS designed the study, extracted the data, and performed all statistical analyses. YoS wrote the draft of the manuscript. WZ reviewed the data analysis and interpretation, and revised the manuscript for the final version.
All the authors gave final approval of the version to be published and agree to be accountable for all aspects of the work regarding questions related to the accuracy or integrity of any part of the work.

Ethics approval and consent to participate

The establishment of this database was approved by the Massachusetts Institute of Technology (Cambridge, MA) and Beth Israel Deaconess Medical Center (Boston, MA) and consent was obtained for the original data collection. Therefore, the ethical approval statement and the need for informed consent were waived for this manuscript.

\section{Consent for publication}

Not applicable.

\section{Competing interests}

The authors declare that they have no competing interests.

\section{Publisher's Note}

Springer Nature remains neutral with regard to jurisdictional claims in published maps and institutional affiliations.

\section{Author details}

'Department of Intensive Care Unit, Zhejiang Hospital, No. 12, Lingyin Road, Hangzhou, Zhejiang 322100, People's Republic of China. 'Department of Intensive Care Unit, Dongyang People's Hospital, No. 60, Wuning West Road, Dongyang, Zhejiang 322100, People's Republic of China. ${ }^{3}$ Department of Breast Surgery, The First Affiliated Hospital, Zhejiang Chinese Medical University, Hangzhou, 310014, China.

Received: 25 May 2018 Accepted: 10 October 2018

Published online: 10 January 2019

References

1. Brotfain E, Koyfman L, Toledano R, Borer A, Fucs L, Galante O, Frenkel A, Kutz R, Klein M. Positive fluid balance as a major predictor of clinical outcome of patients with sepsis/septic shock after discharge from intensive care unit. Am J Emerg Med. 2016:34:2122.

2. Acheampong A, Vincent JL. A positive fluid balance is an independent prognostic factor in patients with sepsis. Crit Care (London, England). 2015;19:251.

3. Wiedemann HP, Wheeler AP, Bernard GR, Thompson BT, Hayden D, deBoisblanc B, Connors AF Jr, Hite RD, Harabin AL. Comparison of two fluid-management strategies in acute lung injury. N Engl J Med. 2006;354(24):2564-75.

4. Payen D, de Pont AC, Sakr Y, Spies C, Reinhart K, Vincent JL. A positive fluid balance is associated with a worse outcome in patients with acute renal failure. Crit Care (London, England). 2008;12(3):R74.

5. Silbert BI, Ho KM, Lipman J, Roberts JA, Corcoran TB, Morgan DJ, Pavey W, Mas $E$, Barden $A E$, Mori TA. Does furosemide increase oxidative stress in acute kidney injury? Antioxid Redox Signal. 2017;26(5):221-6.

6. Ho KM, Sheridan DJ. Meta-analysis of frusemide to prevent or treat acute renal failure. BMJ. 2006;333(7565):420.

7. Bagshaw SM, Gibney RTN, Kruger P, Hassan I, McAlister FA, Bellomo R. The effect of low-dose furosemide in critically ill patients with early acute kidney injury: a pilot randomized blinded controlled trial (the SPARK study). J Crit Care. 2017:42:138-46.

8. Grams ME, Estrella MM, Coresh J, Brower RG, Liu KD. Fluid balance, diuretic use, and mortality in acute kidney injury. Clin J Am Soc Nephrol. 2011;6(5):966-73.

9. de Louw EJ, Sun PO, Lee J, Feng M, Mark RG, Celi LA, Mukamal KJ, Danziger J. Increased incidence of diuretic use in critically ill obese patients. J Crit Care. 2015;30(3):619-23.

10. Jones SL, Martensson J, Glassford NJ, Eastwood GM, Bellomo R. Loop diuretic therapy in the critically ill: a survey. Crit Care Resusc. 2015;17(3):223-6.

11. Johnson AEW, Pollard TJ, Shen L, et al. Scientific Data. 2016. https://doi.org/ 10.1038/sdata.2016.35.

12. Kellum JA, Lameire N. Diagnosis, evaluation, and management of acute kidney injury: a KDIGO summary (Part 1). Crit Care (London, England). 2013; 17(1):204.

13. Lameire N, Kellum JA. Contrast-induced acute kidney injury and renal support for acute kidney injury: a KDIGO summary (Part 2). Crit Care (London, England). 2013;17(1):205. 
14. Zavada J, Hoste E, Cartin-Ceba R, Calzavacca P, Gajic O, Clermont G, Bellomo R, Kellum JA. A comparison of three methods to estimate baseline creatinine for RIFLE classification. Nephrol Dial Transplant. 2010;25(12):3911-8.

15. Zhang Z. Propensity score method: a non-parametric technique to reduce model dependence. Ann Transl Med. 2017;5(1):7.

16. Rhodes A, Evans LE, Alhazzani W, Levy MM, Antonelli M, Ferrer R, Kumar A, Sevransky JE, Sprung CL, Nunnally ME, et al. Surviving Sepsis Campaign: international guidelines for management of sepsis and septic shock: 2016. Intensive Care Med. 2017:43(3):304-77.

17. Butler FK, Holcomb JB, Schreiber MA, Kotwal RS, Jenkins DA, Champion HR, Bowling F, Cap AP, Dubose JJ, Dorlac WC, et al. Fluid resuscitation for hemorrhagic shock in tactical combat casualty care: TCCC guidelines change 14-01-2 June 2014. J Spec Oper Med. 2014;14(3):13-38.

18. Silva JM Jr, de Oliveira AM, Nogueira FA, Vianna PM, Pereira Filho MC, Dias LF, Maia VP, Neucamp Cde S, Amendola CP, Carmona MJ, et al. The effect of excess fluid balance on the mortality rate of surgical patients: a multicenter prospective study. Crit Care (London, England). 2013;17(6):R288.

19. Balogh Z, Moore FA, Moore EE, Biffl WL. Secondary abdominal compartment syndrome: a potential threat for all trauma clinicians. Injury. 2007;38(3):272-9.

20. Coats TJ, Brazil E, Heron M, MacCallum PK. Impairment of coagulation by commonly used resuscitation fluids in human volunteers. Emerg Med J. 2006:23(11):846-9.

21. Boyd JH, Forbes J, Nakada TA, Walley KR, Russell JA. Fluid resuscitation in septic shock: a positive fluid balance and elevated central venous pressure are associated with increased mortality. Crit Care Med. 2011;39(2):259-65.

22. Wang $\mathrm{CH}$, Hsieh WH, Chou HC, Huang YS, Shen $\mathrm{JH}$, Yeo YH, Chang HE, Chen SC, Lee CC. Liberal versus restricted fluid resuscitation strategies in trauma patients: a systematic review and meta-analysis of randomized controlled trials and observational studies. Crit Care Med. 2014;42(4):954-61.

23. Silversides JA, Major E, Ferguson AJ, Mann EE, McAuley DF, Marshall JC, Blackwood B, Fan E. Conservative fluid management or deresuscitation for patients with sepsis or acute respiratory distress syndrome following the resuscitation phase of critical illness: a systematic review and meta-analysis. Intensive Care Med. 2017:43(2):155-70.

24. Slater MB, Gruneir A, Rochon PA, Howard AW, Koren G, Parshuram CS. Identifying high-risk medications associated with acute kidney injury in critically ill patients: a pharmacoepidemiologic evaluation. Paediatr Drugs. 2017;19(1):59-67.

25. Mehta RL, Pascual MT, Soroko S, Chertow GM. Diuretics, mortality, and nonrecovery of renal function in acute renal failure. JAMA. 2002;288(20):2547-53.

26. Wu VC, Lai CF, Shiao CC, Lin YF, Wu PC, Chao CT, Hu FC, Huang TM, Yeh YC, Tsai IJ, et al. Effect of diuretic use on 30-day postdialysis mortality in critically ill patients receiving acute dialysis. PLoS One. 2012;7(3):e30836.

27. Uchino S, Doig GS, Bellomo R, Morimatsu H, Morgera S, Schetz M, Tan I, Bouman C, Nacedo E, Gibney N, et al. Diuretics and mortality in acute renal failure. Crit Care Med. 2004;32(8):1669-77.

28. Dellinger RP, Levy MM, Carlet JM, Bion J, Parker MM, Jaeschke R, Reinhart K, Angus DC, Brun-Buisson C, Beale R, et al. Surviving Sepsis Campaign: international guidelines for management of severe sepsis and septic shock: 2008. Intensive Care Med. 2008;34(1):17-60.

29. Shen $Y$, Huang $X$, Zhang W. Association between fluid intake and mortality in critically ill patients with negative fluid balance: a retrospective cohort study. Crit Care (London, England). 2017;21(1):104.

30. Yeh DD, Tang JF, Chang Y. The use of furosemide in critically ill trauma patients: a retrospective review. J Emerg Trauma Shock. 2014;7(2):83-7.

31. Shann F. Pharmacodynamics of intravenous frusemide bolus in critically ill patients. Crit Care Resusc. 2017;19(4):355.

Ready to submit your research? Choose BMC and benefit from:

- fast, convenient online submission

- thorough peer review by experienced researchers in your field

- rapid publication on acceptance

- support for research data, including large and complex data types

- gold Open Access which fosters wider collaboration and increased citations

- maximum visibility for your research: over $100 \mathrm{M}$ website views per year

At BMC, research is always in progress.

Learn more biomedcentral.com/submissions 